\title{
ON THE CORE OF THE NETWORK DESIGN GAME
}

\author{
Mikio Kubo Hiroshi Kasugai \\ Waseda University
}

(Received May 16, 1991; Revised September 4, 1991)

\begin{abstract}
We consider a cooperative game on a communication network system whose objective is to find a fair allocation of the total cost for constructing the network among customers. Since an NP-hard combinatorial optimization problem must be solved for calculating the exact network design cost, we consider a game in which lower bounds obtained by Lagrangean relaxation are used instead of the exact costs. We derive a fair cost allocation for the game and show that the derived allocation vectors belong to the core.
\end{abstract}

\section{Introduction}

In this paper we consider a cooperative game defined on the network design problem. The network design problem can be seen as a generalization of the minimum spanning tree problem, the Steiner tree problem, the location problem, and other important graph theoretical combinatorial optimization problems, and, more importantly, the network design problem itself has wide applicabability to several practical areas such as urban transportation, communication, production and distribution network models. This implies practical importance of the cost allocation model for the network design problem.

Very limited work has been done for the cost allocation problem defined on the network design problem. In the literature, this class of problems is called the network synthesis game [8] or the network design game [15]. Granot and Hojati [8] analyzed two special cases of the network design game and proved the nonemptiness of the core of these games using the submodular function. Tamir [15] considered a network design game which corresponds to the linear programming relaxation of our problem. He also analyzed Granot and Hojati's special cases. Several special cases of the network design game such as the traveling salesman problem [14], the location problem [5], [16], the minimum spanning tree problem [6], [7], [9], the Steiner tree problem [10] have been paid much attention in the literature. Though several special cases of the network design game are practically important, the general one is also important; we will pay attention to the general class of the network design game.

The remaining part of this paper is organized as follows: In Section 2 we describe the precise definition of the network design cost allocation game and give some preliminaries. In Section 3 we consider the case in which the cost functions are replaced by lower bounds, and derive a fair cost allocations for this modified game. Section 4 contains concluding remarks.

\section{Problem Definition and Preliminaries}

The cost allocation problem associated with the network design problem can be formulated as the network design cost allocation game (NDG) defined as follows: Given a directed graph $G(N, A)$ where $N$ is a set of nodes $\{1, \cdots, n\}$ and $A$ is a set of $\operatorname{arcs}$. Let $K \subseteq N \times N$ be the set of distinct commodities. For each $k \in K$, one unit of commodity must be sent from the origin $O(k) \in N$ to the destination $D(k) \in N-\{O(k)\}$. The total network design cost is the sum of two cost functions: the fixed cost $F_{i j}$ incurred when arc $(i, j)$ is constructed and the variable (routing) cost $d_{i j}^{k}$ incurred when one unit of commodity $k$ passes through $\operatorname{arc}(i, j)$.

The cost incurred to flow the commodities in set $S \subseteq N$ is denoted by $c(S)$ which is the sum of the fixed and variable costs. We consider three cases for computing $c(S)$. 
Case 1: The customer on the node sends and pays for commodities to other nodes.

Case 2: The customer on the node receives and pays for commodities from other nodes.

Case 3: The customer on the node both sends and receives commodities. That is, each commodity is shipped in both directions, and the cost is evenly distributed between the sender and the recipient of the commodity.

In Case 1 (or Case 2), we define $K(S) \subseteq K$ as the set of commodities associated with the coalition $S \subseteq N$ such that $O(k) \in S$ (or $D(k) \in S$ ). In Case 3 , we define $K(S) \subseteq K$ as the set of commodities such that $O(k) \in S$ or $D(k) \in S$ for $S \subseteq N$.

Then the cost of coalition $S$ is obtained by solving the following optimization problem.

$$
c(S)=\min \sum_{(i, j) \in A} \sum_{k \in K(S)} d_{i j}^{k} f_{i j}^{k}+\sum_{(i, j) \in A} F_{i j} y_{i j}
$$

subject to

$$
\begin{gathered}
\sum_{j \in N-\{i\}} f_{i j}^{k}-\sum_{j \in N-\{i\}} f_{j i}^{k}=\left\{\begin{array}{cl}
1 & i=O(k) \\
0 & \forall i \in V-\{O(k), D(k)\} \quad \forall k \in K(S) \\
-1 \quad i=D(k)
\end{array} \quad \forall(i, j) \in A\right. \\
\sum_{k \in K(S)} f_{i j}^{k} \leq|K(S)| y_{i j} \quad \forall(i, j) \in A, k \in K(S) \\
f_{i j}^{k} \geq 0 \quad \forall(i, k) \in A \\
y_{i j} \in\{0,1\} \quad \forall(i, j) \in A
\end{gathered}
$$

In the formulation above, the objective function (1) minimizes the total cost consisting of the sum of the fixed and variable costs. Constraints (2) are the flow conservation equations. Constraints (3) represent that the flow volume thorough arc $(i, j)$ must be 0 if the arc is not constructed. The network design game (NDG) can be represented by $(N ; c)$ where $N$ and $c$ represents the set of players and the characteristic function, respectively.

Remark that the traveling salesman game [14] is a variant of the NDG with the additional 'degree' constraint, the Steiner tree and minimum spanning tree games [6], [7], [9], [10] are special cases of the NDG in which the source of the commodities is only one node, and the location game [5], [16] can be seen as the NDG on a specially structured network.

The problem we are considering is to allocate the total cost $c(N)$ to the set $N$ of players. Among many fair allocation schemes for the cooperative game, we use the following concept.

Definition 1 A core of the game $(N ; c)$ is the set of all vectors $x=\left(x_{1}, x_{2}, \cdots, x_{n}\right)$ such that

$$
\sum_{i \in S} x_{i} \leq c(S) \quad \forall S \subset N
$$

and

$$
\sum_{i \in N} x_{i}=c(N)
$$


We easily observe that the cost function of Case 1 (or Case 2) does not exceed that of Case 3. This leads that if the core for Case 1 (or Case 2) is non-empty, so is Case 3; in the sequel we concentrate on Case 1 in which the player on node acts as a sender of commodities and the set of commodities $K(S)$ for a coalition $S \subseteq N$ is defined by $K(S)=$ $\{k \in K \mid O(k) \in S\}$. Of course our results can be extended to Case 2 and Case 3 without any difficulty.

Several researches have been analyzed the NDG and derive some sufficient conditions for the problem having the core [8], [15]. Unfortunately, Megiddo [10] gave an example in which the core of the Steiner tree game is empty (see also Tamir [15]). Since the Steiner tree problem is a special case of the network design problem, the NDG may have an empty core, i.e., there may not exist such a fair allocation for the NDG. Since the network design problem is proved to be NP-hard [2], major developments for the algorithmic approach to solve the network design problem concentrate on the lower bounding procedures or the heuristic algorithms. Similarly since the NDG is very likely to hard to solve, and, furthermore, computing $c(N)$ itself is an essentially difficult task, we use the lower bounds of the characteristic function of the NDG. For coalition $S(\subseteq N)$, let $\bar{c}(S)$ be the lower bound of $c(S)$. In the following section, we analyze the game $(N ; \bar{c})$ called the lower bound NDG.

\section{Core of Lower Bound Network Design Game}

In this section we consider $(N ; \bar{c})$ in which lower bounds are used instead of using the exact cost for constructing the network. A naive technique to derive lower bounds of the network design problem is the linear programming relaxation. If we replace constraints (5) with the following constraints

$$
0 \leq y_{i j} \leq 1 \quad \forall(i, j) \in A
$$

we obtain the linear programming relaxation of the network design problem. Further we can easily verify that the linear programming relaxation satisfies the additivity assumption of the linear production game analyzed by Owen [11]. Therefore we can get a core allocation vector using the dual price of the relaxed problem. Of course, in this case, the core is not empty as shown in Tamir [15]. This fact is a direct consequence of Owen's linear production game [11].

Unfortunately the linear programming relaxation does not give tight lower bounds. The lower bound which is far below the original cost is not useful in practice. Another practical procedure for getting lower bounds is the Lagrangean relaxation which sometimes gives tighter lower bounds if we select the relaxed constraints carefully; next we apply the Lagrangean relaxation.

Given a vector of Lagrangean multipliers $v=\left[v_{i}^{k}\right]$, we can get the following Lagrangean relaxation problem.

\section{(LRP : Lagrangean Relaxation Problem)}

$$
L(S, v)=\min \sum_{(i, j) \in A} \sum_{k \in K(S)}\left(d_{i j}^{k}+v_{i}^{k}-v_{j}^{k}\right) f_{i j}^{k}+\sum_{(i, j) \in A} F_{i j} y_{i j}+\sum_{k \in K(S)} v_{D(k)}^{k}
$$

subject to $(3),(4),(5)$.

The Lagrangean lower bound $\bar{c}(S)$ for coalition $S$ can be obtained by calculating the best bound derived by the LRP. More formally the Lagrangean lower bound $\bar{c}(S)$ of the cost function $c(S)$ can be obtained as follows:

$$
\bar{c}(S)=\max _{v} L(S, v) .
$$


The maximum lower bound can be obtained by the subgradient optimization technique (see for example [13]), and the derived bound can be proved to be at least better than the one obtained by the linear programming relaxation.

Since the equations (2) are the only constraints that connect all arcs, by dualizing this constraints, we obtain the subproblems for each arc which can be be solved independently. The optimal solution $(\tilde{f}(S), \tilde{y}(S))$ of the LRP can be obtained as follows:

$$
\tilde{y}_{i j}(S)=\left\{\begin{array}{cc}
1 & \text { if } \sum_{k \in K(S)}\left(-d_{i j}^{k}-v_{i}^{k}+v_{j}^{k}\right)^{+}>F_{i j} \\
0 & \text { otherwise }
\end{array} \quad \forall(i, j) \in A\right.
$$

and

$$
\tilde{f}_{i j}^{k}(S)=\left\{\begin{array}{cc}
\tilde{y}_{i j}(S) & \text { if } d_{i j}^{k}+v_{i}^{k}-v_{j}^{k}<0 \\
0 & \text { otherwise }
\end{array} \quad \forall(i, j) \in A, k \in K(S)\right.
$$

where $(a)^{+}$represents $\max \{a, 0\}$.

Then we get the following theorem.

Theorem 1 Let the optimal dual vector for the grand coalition $N$ be $\hat{v}$ and the optimal solution of the LRP for the grand coalition $N$ be $(\tilde{f}(N), \tilde{y}(N))$. We define

$$
x_{i}=\sum_{O(k)=i}\left[\hat{v}_{D(k)}^{k}+\sum_{(i, j) \in \hat{A}}\left\{\delta_{i j}^{k} \tilde{f}_{i j}^{k}(N)+\frac{\delta_{i j}^{k}}{\Delta_{i j}} F_{i j} \tilde{y}_{i j}(N)\right\}\right]
$$

where $\delta_{i j}^{k}=d_{i j}^{k}+\hat{v}_{i}^{k}-\hat{v}_{j}^{k}, \Delta_{i j}=\sum_{k \in K}\left(d_{i j}^{k}+\hat{v}_{i}^{k}-\hat{v}_{j}^{k}\right) \tilde{f}_{i j}^{k}(N)$ and $\hat{A}=\left\{(i, j) \in A \mid \Delta_{i j}<0\right\}$. Then the vector $x$ belongs to the core of $(N ; \bar{c})$.

Proof : Obviously we get $\bar{c}(N)=\sum_{i \in N} x_{i}$. The remaining problem is to prove $\bar{c}(S) \geq$ $\sum_{i \in S} x_{i}$ for all $S \subset N$. From the construction of the Lagrangean dual, we get

$$
\begin{aligned}
\bar{c}(S) & =\max _{v} L(S, v) \\
& \geq L(S, \hat{v}) \\
& =\sum_{k \in K(S)} \hat{v}_{D(k)}^{k}+\sum_{(i, j) \in A} \sum_{k \in K(S)} \delta_{i j}^{k} \tilde{f}_{i j}^{k}(S)+\sum_{(i, j) \in A} F_{i j} \tilde{y}_{i j}(S),
\end{aligned}
$$

where $(\tilde{f}(S), \tilde{y}(S))$ is the optimal solution vector of the LRP for the coalition $S$. From the construction of the vector $x$, we get

$$
\begin{aligned}
\sum_{i \in S} x_{i} & =\sum_{i \in S} \sum_{O(k)=i}\left[\hat{v}_{D(k)}^{k}+\sum_{(i, j) \in \hat{A}}\left\{\delta_{i j}^{k} \tilde{f}_{i j}^{k}(N)+\frac{\delta_{i j}^{k}}{\Delta_{i j}} F_{i j} \tilde{y}_{i j}(N)\right\}\right] \\
& =\sum_{k \in K(S)} \hat{v}_{D(k)}^{k}+\sum_{(i, j) \in \hat{A}} \sum_{k \in K(S)} \delta_{i j}^{k} \tilde{f}_{i j}^{k}(N)+\sum_{(i, j) \in \hat{A}} \sum_{k \in K(S)} \frac{\delta_{i j}^{k}}{\Delta_{i j}} F_{i j} \tilde{y}_{i j}(N) .
\end{aligned}
$$

Below we show $\bar{c}(S) \geq \sum_{i \in S} x_{i}$ by comparing each term in (14) and (15). The first term is identical. Since we restrict the problem on node set $S$, we get $\tilde{f}_{i j}^{k}(S) \leq \tilde{f}_{i j}^{k}(N)$ for all $(i, j) \in A, k \in K, S \subseteq N$. This implies that the second term in (15) is not larger than in (14). To compare the third term we consider three cases.

1. When $\tilde{y}_{i j}(N)=0$ :

In this case, obviously, $\tilde{y}_{i j}(S)=0$; the third term is identical. 
2. When $\tilde{y}_{i j}(N)=\tilde{y}_{i j}(S)=1$ :

Obviously the third in (15) is not larger than the one in (14).

3. When $\tilde{y}_{i j}(N)=1$ and $\tilde{y}_{i j}(S)=0$ :

In this case we must compare the sum of the second term and the third term. First we get $\tilde{f}_{i j}^{k}(S)=0$ for all $k \in K$ because $\tilde{y}_{i j}(S)=0$. This implies that the sum of the second and third terms in (14) is equal to 0 in this case. Below we prove that the sum of the second and third terms in (15) is not greater than 0 . By the procedure to solve the LRP, we get $\tilde{f}_{i j}^{k}(N)=1$ if $\delta_{i j}^{k}<0$ if $\tilde{y}_{i j}=1$. For each $\operatorname{arc}(i, j) \in \hat{A}$ and $k \in K(S)$ such that $\tilde{f}_{i j}^{k}(N)=1, \delta_{i j}^{k}+\frac{\delta_{i j}^{k}}{\Delta_{i j}} F_{i j}=\delta_{i j}^{k}\left(1+\frac{F_{i j}}{\Delta_{i j}}\right)<0$ because

$$
\begin{aligned}
\left|\Delta_{i j}\right| & =\sum_{k \in K(N)}\left(-d_{i j}^{k}-v_{i}^{k}+v_{j}^{k}\right)^{+} \\
& \geq \sum_{k \in K(S)}\left(-d_{i j}^{k}-v_{i}^{k}+v_{j}^{k}\right)^{+} \\
& >F_{i j} .
\end{aligned}
$$

The last strict inequality follows from $(11)$ and $\tilde{y}_{i j}(S)=1$. This, by combining $\delta_{i j}^{k}<0$, implies the desired result.

By combining the above results, we get $\bar{c}(S) \geq \sum_{k \in K(S)} x_{k}$. I

Once the Lagrangean lower bound can be obtained using the subgradient method [13] or the dual ascent procedure proposed by Balakrishnan, Magnanti and Wong [1], we get a cost allocation vector $x$ using the formula (13) easily; thus the computational requirement for computing the allocation vector is moderate.

Next we turn to the analysis the relations among the lower bounds obtained by Lagrangean relaxation and by linear programming relaxation, and the original costs.

Proposition 1 For coalition $S \subseteq N$, let $c(S), \vec{c}(S)$, and $\bar{c}_{L P}(S)$ be the optimal cost function, the cost function obtained by Lagrangean relaxation, and the cost function obtained by linear programming relaxation, respectively. Then the following relations hold.

$$
\bar{c}_{L P}(S) \leq \bar{c}(S) \leq c(S)
$$

Proof: Since the linear programming and Lagrangean problems relax some of constraints of the original network design problem, we get $\bar{c}_{L P}(S) \leq c(S)$ and $\bar{c}(S) \leq c(S)$. Since the linear programming relaxation of the LRP does not necessarily give an integer solution, we get, from the duality theory $[3], \bar{c}_{L P}(S) \leq \bar{c}(S)$.

Balakrishnan, Magnanti and Wong [1] show that Lagrangean lower bounds obtained by the dual ascent procedure are guaranteed to be within $1-3 \%$ of optimality in practice. Thus we get a very tight lower estimate of the core using the Lagrangean relaxation technique.

\section{Concluding Remarks}

We have derived cost allocation vectors in the core for the network design game in which cost functions are replaced by the lower bounds obtained by Lagrangean relaxation technique. The network design problem involves several famous problems such as the location problem, the Steiner tree problem, the production lot-size problem; thus our results can be applied to such problems. Further the network design problem itself has wide applicability to several design problems such as the distribution network design, the communication network design, the transportation network design; our results are of use for allocating the total costs to construct network systems in such practical situations. 


\section{References}

[1] A. Balakrishnan, T. L. Magnanti and R. T. Wong ,"A Dual-Ascent Procedure for Large-Scale Uncapacitated Network Design ," Operations Research, Vol. 37 (1989) pp. 716-740.

[2] M. R. Garey and D. S. Johnson,"Computers and Intractability: A Guide to the Theory of NP-Completeness," Freeman (1979).

[3] A. M. Geoffrion,"Lagrangean Relaxation for Integer Programming," Mathematical Programming Study, Vol. 2 (1974) pp. 82-114.

[4] D. Granot," A Generalized Linear Production Model: A Unifying Model," Mathematical Programming, Vol. 34 (1986) pp. 212-222.

[5] D. Granot,"On the Role of Cost Allocation in Locational Models," Operations Research, Vol. 35 (1987) pp. 234-248.

[6] D. Granot and G. Huberman,"Minimum Cost Spanning Tree Game," Mathematical Programming, Vol. 21 (1981) pp.1-18.

[7] D. Granot and G. Huberman,"On the Core and Nucleolus of M.C.S.T. Games," Mathematical Programming, Vol. 29 (1984) pp.323-347.

[8] D. Granot and M. Hojati,"On Cost Allocation in Communication Network," Networks, Vol. 20 (1990) pp. 209-229.

[9] N. Megiddo,"Computational Complexity and the Game Theory Approach to Cost Allocation for a Tree," Mathematics of Operations Research, Vol.3 (1978) pp. 189-196.

[10] N. Megiddo,"Cost Allocation for Steiner Tree, "Networks, Vol. 8 (1978) pp. 1-6.

[11] G. Owen,"On the Core of Linear Production Games," Mathematical Programming, Vol. 9 (1975) pp. 358-370.

[12] L. S. Shapley and L. K. Raut," Theory of Games and its Application to Economics and Policies," ISI Lecture Note, No. 10, Macmillan India (1981).

[13] N. Z. Shor ,"Minimization Methods for Nondifferentiable Functions," Springer-Verlag (1985).

[14] A. Tamir,"On the Core of a Traveling Salesman Cost Allocation Game," Operations Research Letters, Vol. 8 (1989) pp. 31-34.

[15] A. Tamir,"On the Core of Network Synthesis Games," Mathematical Programming, Vol. 50 (1991) pp. 123-135.

[16] A. Tamir,"On the Core of Cost Allocation Games Defined on Location Problems," to appear in Transportation Science (1991).

Mikio KUBO : Department of Industrial Engineering and Management, Waseda University, 3-4-1, Okubo Shinjuku, Tokyo 169, Japan 